\title{
Mutations in KaiA, a clock protein, extend the period of circadian rhythm in the cyanobacterium Synechococcus elongatus PCC 7942
}

\author{
Hideya Nishimura, ${ }^{1,2} \dagger$ Yoichi Nakahira, ${ }^{1} \dagger$ Keiko Imai, ${ }^{1}$ Akiko Tsuruhara, \\ Hisayo Kondo, ${ }^{1}$ Hiroshi Hayashi, ${ }^{2}$ Makoto Hirai, ${ }^{2}$ Hidehiko Saito ${ }^{2}$ \\ and Takao Kondo ${ }^{1}$
}

Author for correspondence: Takao Kondo. Tel: +8152789 2498. Fax: +81527892963. e-mail: kondo@bio.nagoya-u.ac.jp

1 Division of Biological Science, Graduate School of Science, Nagoya University and CREST, Japan Science and Technology Corporation (JST), Furo-cho, Chikusa, Nagoya 464-8602, Japan

2 First Department of Internal Medicine, Nagoya University, School of Medicine Tsurumai 65 Showa, Nagoya 466-8550, Japan

\begin{abstract}
KaiA KaiB and KaiC are essential circadian clock proteins in the unicellular cyanobacterium Synechococcus elongatus PCC 7942. KaiA protein activates transcription of the kaiBC operon, which is believed to be a crucial step in the oscillating feedback loop of cyanobacteria. In this study, 400 mutations were introduced into kaiA by PCR-based mutagenesis, and rhythmic phenotypes of these mutants were studied by a bioluminescence reporter. In contrast to mutations in KaiB or KaiC, the vast majority of KaiA mutations extended the period and only rarely shortened it. The period could be extended to $35 \mathrm{~h}$ without lowering the mean or peak levels of kaiBC expression. However, several mutations resulted in low-amplitude oscillations or arrhythmia, which were accompanied by lowered kaiBC transcription. These results imply that the KaiA protein can change the period length of the circadian rhythm directly (through an unknown biochemical mechanism) or indirectly (by lowering kaiBC expression). Specific mutations of KaiA were identified in 34 mutants. While mutations mapped to various locations of the KaiA sequence, two clusters of period-altering mutations were found. This suggested that these regions are important domains of the KaiA protein for defining the period length. On the other hand, different sequences within KaiA to which arrhythmic mutations were mapped are important to enhance kaiBC expression.
\end{abstract}

Keywords: circadian clock, kai genes, kaiBC expression, amplitude of rhythm, bioluminescence rhythm

\section{INTRODUCTION}

Circadian rhythms, biological oscillations with a period of approximately $24 \mathrm{~h}$, have been found in many diverse organisms, ranging in complexity from bacteria to green plants and mammals. These rhythms regulate various metabolic and behavioural activities and are believed to be adaptable to daily changes in environmental conditions, such as light, temperature and humidity (Bünning, 1973; Pittendrigh, 1993). The circadian clock, an intracellular mechanism that generates oscillations with $\sim 24 \mathrm{~h}$ periods, has been postulated as a basis for these

†These authors contributed equally to this work.

Abbreviations: LL, constant illumination; PMT, photomultiplier tube. rhythms, while feedback models of molecular cycling have been proposed for Neurospora, Drosophila and mammals (Dunlap, 1999; Young \& Kay, 2001). In these models, clock protein interactions function as crucial steps in feedback loops with a circadian time-course.

Cyanobacteria are the simplest organisms that display circadian rhythms (Golden et al., 1998; Kondo \& Ishiura, 2000; Iwasaki \& Kondo, 2000). We have previously introduced a bioluminescence reporter gene into the unicellular cyanobacterium Synechococcus elongatus PCC 7942 to monitor circadian gene expression (Kondo et al., 1993), isolated various clock mutants (Kondo et al., 1994), and both identified and cloned the clock gene cluster kaiABC, composed of kaiA, kaiB and kaiC (Ishiura et al., 1998). While no 
similarity was found between Kai proteins and the clock proteins of other organisms, disruption of any single kai gene completely abolished circadian rhythm. Experiments in which kai genes are overexpressed suggest that KaiC represses its own (kaiBC) expression while KaiA enhances it. Thus, KaiC and KaiA have been proposed to be negative and positive elements, respectively, in the molecular feedback loop of kaiBC expression (Ishiura et al., 1998).

Nineteen distinct mutants exhibiting a wide range of period lengths and arrhythmia mapped their mutations onto the kaiC gene (Ishiura et al., 1998). In light of this, it seems highly likely that understanding the biochemistry of the $\mathrm{KaiC}$ protein will be key in understanding circadian characteristics. Previous studies have shown that KaiA and KaiC interact with each other in the yeast two-hybrid system, in vitro, and in Synechococcus; closer examination revealed that the two half-units of KaiC (CI and CII, the first and second half domains, respectively) both interact with KaiA (Iwasaki et al., 1999). The ATP-binding and autophosphorylation capacities of KaiC have also been shown to be important parts of the circadian timing mechanism (Nishiwaki et al., 2000). Furthermore, two KaiA-binding domains (CKABD1 and CKABD2) in KaiC were found on the Cterminal regions of $\mathrm{CI}$ and $\mathrm{CII}$, respectively, by using the yeast two-hybrid system and an in vitro interaction assay (Taniguchi et al., 2001). More recently, SasA, a regulatory element consisting of a two-component sensory kinase, has been found to amplify the kai-based oscillations through its interaction with KaiC (Iwasaki et al., 2000).

In previous work (Ishiura et al., 1998), only four KaiA mutants were identified after chemical mutagenesis. While the phenotypes of these mutants include arrhythmia, which indicates that KaiA is crucial in the establishment or maintenance of circadian rhythm, further mutational analysis is necessary to understand the function of KaiA. In this study, we performed PCRbased mutagenesis on the kaiA gene and screened for abnormal rhythmic phenotypes. Using targeting of gene integration, followed by high throughput screening for rhythmic phenotypes with bioluminescence, we obtained $\sim 400$ mutants and analysed their phenotypes. Point mutations of representative kaiA mutants were also identified and mapped onto the KaiA protein. These mutations were concentrated in two distinct regions. We discuss the implications of our findings on the potential role of KaiA in generating circadian oscillations in cyanobacteria.

\section{METHODS}

Cyanobacteria strains and culture conditions. Synechococcus elongatus PCC 7942 was used as the background strain in this study. All Synechococcus strains were cultured in either modified BG-11 liquid medium or solid BG-11 medium containing $1.5 \%$ Bacto agar (Bustos \& Golden, 1991) under constant illumination (LL) of $46 \mu \mathrm{mol} \mathrm{m} \mathrm{m}^{-2} \mathrm{~s}^{-1}$ from white fluorescent lamps at $30^{\circ} \mathrm{C}$. NUC42 and NUC43 are the wild-

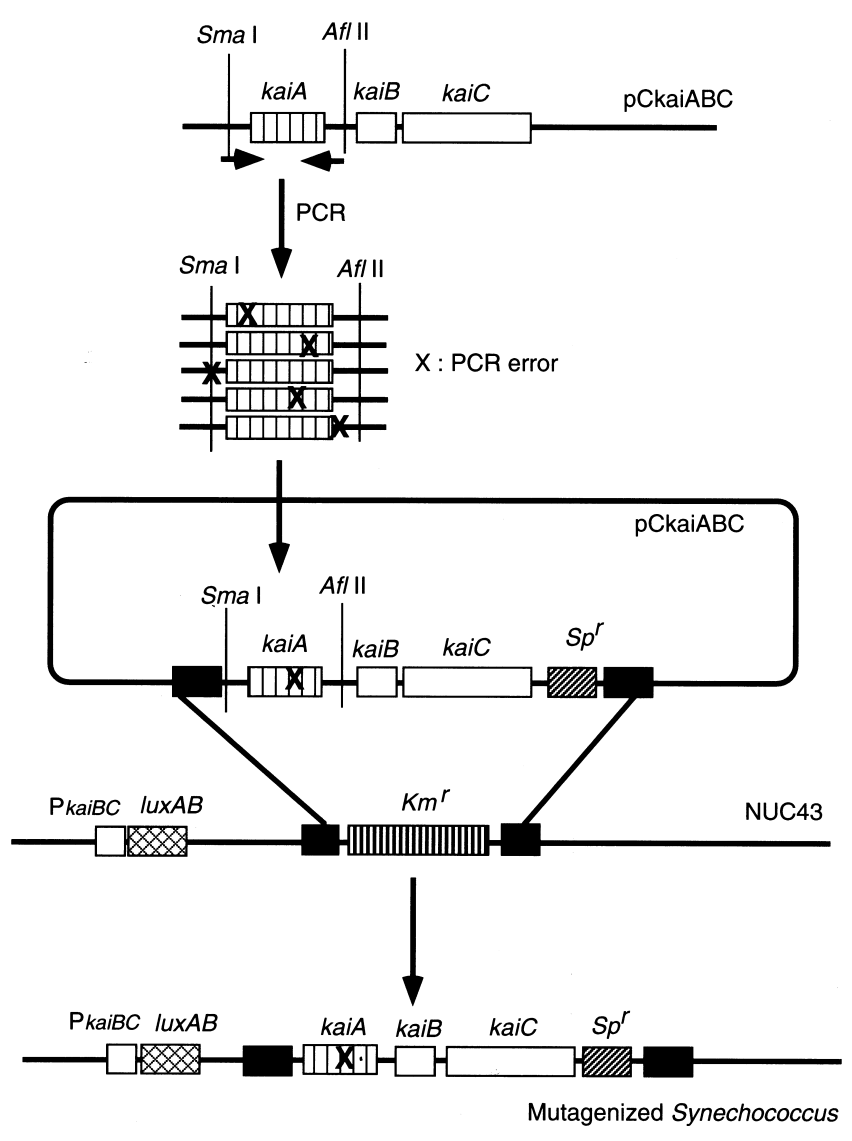

Fig. 1. Introduction of mutations into kaiA by $P C R$ error. Synechococcus DNA was digested with Smal and AfIII to obtain a DNA segment that contains the entire kaiA sequence. A PCR reaction was performed to amplify this DNA. PCR errors (labelled $X$ ) were introduced into DNA copies. At the same time, the $\mathrm{pCkai} A B C$ plasmid containing the whole kaiABC operon was digested by Smal and AfIII, to obtain kaiA-deleted DNA. The PCR product and the kaiA-deleted plasmid were fused to obtain kaiABC operons that carry an error-prone PCR product of kaiA. Fused pCkaiABC was introduced into NUC43 Synechococcus to obtain kaiA mutants that could be screened by a CCD camera system.

type and kaiABC-deleted strains, respectively. Both strains carry the PkaiBC:: $\operatorname{lu} x A B$ reporter construct at neutral site I (S. Kutsuna, M. Ishiura \& T. Kondo, unpublished results). The construct is a fusion of a region upstream of $k a i B(-91$ to +6 ) and a promoterless $\ln x A B$ gene set that reports expression of the kaiBC operon. Four kaiA mutations previously obtained by chemical mutagenesis (EMS, ethylmethane sulfonate; Ishiura et al., 1998) are also listed in Fig. 4. Kanamycin-resistant and spectinomycin-resistant transformed clones were selected with $40 \mu \mathrm{g}$ kanamycin or spectinomycin $\mathrm{ml}^{-1}$. Escherichia coli strains DH5 $\alpha$ and DH10B were used as hosts for plasmid construction.

PCR-based mutagenesis of kaiA and construction of mutant strains. PCR-based mutagenesis is a well-documented method in bacteria (Diaz et al., 1991) including a cyanobacterium, Synechocystis sp. PCC 6803 (Narusaka et al., 1999). Fig. 1 illustrates the method of constructing kaiA mutants by PCRbased mutagenesis. pCkaiABC is a targeting plasmid carrying the kaiABC gene and an omega fragment (Prentki \& Krisch, 
1984). Using rTaq DNA polymerase (Takara Shuzo), the kaiA gene was amplified by PCR, using pCkaiABC as a template and two oligonucleotides [B48-up (5'-AACCAATAGGCCGAAATCGGC-3') and 715(1) low (5'-TTGAAGTTCGCCGTAGAGTA-3') (i.e. nt +1068 to +1087 of kaiA)] as primers. The PCR cycle was set at $94{ }^{\circ} \mathrm{C}(30 \mathrm{~s}), 54{ }^{\circ} \mathrm{C}(30 \mathrm{~s}), 72{ }^{\circ} \mathrm{C}$ ( 2 min extension phase) and repeated 30 times. Because Taq DNA polymerase aberrantly recognizes DNA sequences at a constant rate, thereby making incorrect PCR products, some PCR-amplified products of kaiA will have a point mutation at a random location (represented by an $X$ in Fig. 1). The resulting products were digested by $S m a \mathrm{I}$ and $A f l \mathrm{II}$ and ligated to SmaI/AflII-digested pCkaiABC with T4 DNA ligase. NUC43 was obtained by transforming the wild-type reporter strain NUC42 with pDkaiABC (Ishiura et al., 1998), which carries three segments in tandem: a region upstream of the kaiA gene, a kanamycin-resistance gene and a region downstream of the kaiC gene. NUC43 was then transformed with the ligated fusion plasmids. Synechococcus carrying the PCR mutations were selected with spectinomycin and screened for rhythmic profiles.

Bioluminescence rhythm assay. Transformants were cultured on solid BG-11 plates and bioluminescence rhythms were then monitored with a cooled-CCD camera system (Kondo et al., 1994). In total, $\sim 4000$ clones were screened and the period for each clone was calculated. As a more precise assay, bioluminescence profiles of selected mutants were monitored using a photomultiplier tube (PMT)-based system (Ishiura et al., 1998). Synechococcus mutants were grown on solid BG-11 plates for 2-3 days in LL and then subjected to darkness for $12 \mathrm{~h}$ to synchronize the clock. After the $12 \mathrm{~h}$ darkness period, a small dish containing $\mathrm{n}$-decanal (which had been dissolved in vacuum pump oil at a concentration of $3 \%, v / v$ ) was placed on each plate and bioluminescence was monitored under LL at the standard conditions $\left(46 \mu \mathrm{mol} \mathrm{m} \mathrm{m}^{-2} \mathrm{~s}^{-1}\right.$ from white fluorescent lamps at $30^{\circ} \mathrm{C}$ ). In this report, amplitudes of the rhythm were classified into four types (high amplitude, medium amplitude, low amplitude and arrhythmic) by peakto-trough ratio of bioluminescence intensity.

DNA sequence of mutants. We directly sequenced mutant kaiA genes after in vitro amplification of kaiA segments by PCR. Synechococcus cells were mixed in a tube with $0.1 \mathrm{~mm}$ zircon/silica beads and lysed by shaking with a Multi-Beads Shocker (Yasui Kikai). Genomic DNA was extracted by phenol extraction and purified by ethanol precipitation. Segments carrying kaiA were amplified by PCR using the following pair of primers : 818(3) up (5' - ACCGGCCACGTAGGGCTGTCC-3') (-409 to -389 of kaiA) and 715(1)low (described above). DNA sequence was then analysed with a DNA sequence kit and an ABI PRISM model 310 sequencer.

\section{RESULTS AND DISCUSSION}

\section{Efficient mutagenesis by error-prone PCR}

In this study, we obtained $\sim 4000$ Synechococcus clones carrying a PCR copy of the kaiA gene, which were screened with a luciferase reporter for individual circadian rhythms. The luciferase reporter, driven by the kaiBC operon promoter, can indicate transcriptional activity of kaiBC, a central step in the circadian pacemaker of cyanobacteria. The profile of this reporter in the wild-type strain reveals a robust rhythm with a period of $25 \mathrm{~h}$ (under current screening conditions) with a sharp expression peak at the subjective dusk and almost completely shut off at the trough phase (Ishiura et al., 1998). Thus, high-amplitude clones with periods close to that of wild-type (24-26 h) presumably carry wild-type kaiA (i.e. a correct PCR product), a silent mutation (resulting in no change at the amino acid level) or insignificant amino acid alterations. As seen in Table 1 , these clones amount to $\sim 87 \%$ of the total. Nevertheless, we obtained $\sim 400$ mutants in this study, or about $12 \%$ of the total number of clones screened. These mutant clones showed distinct abnormalities in circadian profile, including long periods, low amplitudes and arrhythmia. Sufficient numbers of mutants were obtained to allow statistical analysis of the mutations. Whilst it is difficult to estimate the number of indistinguishable, covert mutations among the clones with normal phenotypes, the error-prone PCR mutation rate can be estimated to be even higher than $12 \%$, indicating that PCR mutagenesis is much more efficient than chemical mutagenesis.

As screening of circadian rhythm inevitably requires normal clone growth, we were only able to screen for regularly growing mutant clones on agar plates. Thus, it is possible that some clones with growth-inhibiting mutations had been lost prior to screening, which would in turn disturb our statistical analysis. However, this is unlikely to be the case here because Synechococcus growth does not change even with the complete deletion of kaiA (Ishiura et al., 1998). If fact, we did not notice any change in growth rate in a wide spectrum of mutant phenotypes obtained in this study. Note also that the complete deletion of kaiA induced complete arrhythmicity and severe lowering of kaiBC expression (Ishiura et al., 1998).

\section{KaiA mutations extend the period}

As shown in period histograms (Fig. 2A and B), the most striking feature of kaiA mutagenesis is an almost complete lack of short-period mutations. We found 301 long period mutants (period $>27 \mathrm{~h}$ ) and 92 arrhythmic mutants, whereas only one mutant showed a shortperiod phenotype and the oscillation rapidly damped in this mutant. Based on these statistical results, it can be concluded that mutations in kaiA generally extend the period, whereas periods can be both extended and shortened by mutations in kaiC (Ishiura et al., 1998) and kaiB (our unpublished data). This tendency also holds true for the four kaiA mutants obtained by chemical (EMS) mutagenesis (Ishiura et al., 1998). The one exceptional mutant (F224S) that showed a short period phenotype of $22 \mathrm{~h}$ (Fig. 3C) is of particular interest; the biochemical properties of this mutant KaiA protein must be analysed to shed further light on the mechanism underlying the cyanobacterial oscillator.

Some KaiA mutants had period lengths as long as $50 \mathrm{~h}$ and many arrhythmic mutants were also found. However, when limited to high-amplitude mutants (peak-totrough ratio $>10$ ), the longest period was about $35 \mathrm{~h}$, a $40 \%$ extension of the $25 \mathrm{~h}$ wild-type period (Fig. 2A). Parameters of the rhythmicity other than period length 
Table 1. Summary of PCR-based mutagenesis of kaiA

\begin{tabular}{|lrccc|}
\hline Class of mutant* & \multicolumn{2}{c}{ No. colonies } & No. mutants & $\begin{array}{c}\text { Percentage of } \\
\text { mutants }\end{array}$ \\
\cline { 2 - 3 } & Total & Period $>\mathbf{2 7} \mathbf{h} \dagger$ & & \\
\hline High amplitude & 3648 & 162 & 162 & $4 \cdot 1$ \\
Low amplitude & 226 & 139 & 226 & $5 \cdot 7$ \\
Arrhythmic & 92 & - & 92 & $2 \cdot 3$ \\
Total & 3966 & - & 480 & $12 \cdot 1$ \\
\hline
\end{tabular}

*Based on amplitude of rhythm, each rhythmic phenotype was classified into three classes. Amplitude is defined as peak-to-trough ratio of bioluminescence intensity. High amplitude, peak-to-trough $>10$; low amplitude, $10>$ peak-to-trough $>3$; arrhythmic, peak-to-trough $<3$.

† Numbers of clones which have a period longer than $27 \mathrm{~h}$.

were not altered in mutants shown in Fig. 3A; the waveform of these mutants is similar to that of wildtype, bioluminescence drops to completely shut off levels at the trough phase and the peak bioluminescence intensity levels were changed only slightly $(-20 \%$ to $+10 \%$ of wild-type expression). In addition, the phase of the rhythm reset by $12 \mathrm{~h}$ darkness was also not altered by the mutations (Fig. 3A). Thus, these KaiA mutations apparently changed the period length selectively, implying a direct role for KaiA in period determination.

Medium-amplitude mutants also sustained robust rhythmicity with period lengths of up to $40 \mathrm{~h}$. As shown in Fig. 3B, the peak kaiBC expression was again little changed $(-20 \%$ to $+10 \%)$, whilst $10-30 \%$ of peak kaiBC expression levels remained at trough phases. Thus, as with the high-amplitude group (Fig. 3A), period extension up to $40 \%$ can be induced without a large change in kaiBC expression. These observations imply that the role of the KaiA protein is to control the period length of the circadian oscillation without activation of kaiBC expression. Direct examination of the biochemical activity of KaiA should be performed to address the molecular mechanism for this periodicity modulation. The phase of rhythm, once reset by $12 \mathrm{~h}$ darkness, was again not altered by these mutations (Fig. 3B).

\section{KaiA mutations lower kaiBC expression}

The periods of lower amplitude mutants spanned an even wider range than those in the high-amplitude group, with period lengths as long as $50 \mathrm{~h}$ (Fig. 2B). Severe low-amplitude mutants (peak-to-trough $<3$ ) and arrhythmic mutants are shown in Fig. 3C. Low-amplitude mutants in Fig. 3C still sustain stable rhythms with $24-50 \mathrm{~h}$ periods, whereas PkaiBC activity was considerably elevated at the trough phase and kaiBC expression at peak phase was lowered by $30-50 \%$. Exceptionally, kaiBC expression was elevated in the A245D mutant but this mutant failed to extend the period length. Mutants with very long periods are shown in Fig. 3D. Note that kaiBC expression was significantly lowered in these very long-period mutants. Thus, the long periods of these mutations were induced not only as a direct effect of altered KaiA biochemistry, but also as an indirect effect of lowering kaiBC expression.

Arrhythmic mutants displayed various profiles. Some mutants showed normal peak and troughs during the first cycle, but were then damped through elevation of the trough level. Other mutants were arrhythmic from the beginning of LL. An important feature of these mutants is that PkaiBC activity was significantly lowered; in general, the level of PkaiBC reporter expression was lower than $10 \%$ of wild-type. As KaiA is known to enhance kaiBC expression (Ishiura et al., 1998), it is likely that the mutant KaiA proteins in this group could not sustain rhythmicity because they fail to activate kaiBC expression.

In this study, we have quantitatively examined the PkaiBC activity of KaiA mutants by normalizing bioluminescence data with colony size and number. From these assays, we found that most KaiA mutations lowered PkaiBC activity to varying degrees. As random mutation generally lowers the native function, these results would be consistent with a role for KaiA in enhancing PkaiBC activity (Ishiura et al., 1998). On the other hand, it appears that the level of kaiBC expression contributes little to period determination, but is important in maintaining robustness of the circadian rhythm.

Note also that the phase of the rhythm reset by $12 \mathrm{~h}$ darkness was changed in some low-amplitude mutants (Fig. 3C; D136V, D136Y and A245D). This suggests that KaiA is also involved in the phase-resetting mechanism of the cyanobacterial clock.

\section{Clustering of mutations on the Kai map}

We subsequently sequenced 45 mutants and found that 39 mutants have a single point mutation while five mutants overlapped with other mutants. Each point mutation was mapped to a residue in KaiA. As illus- 


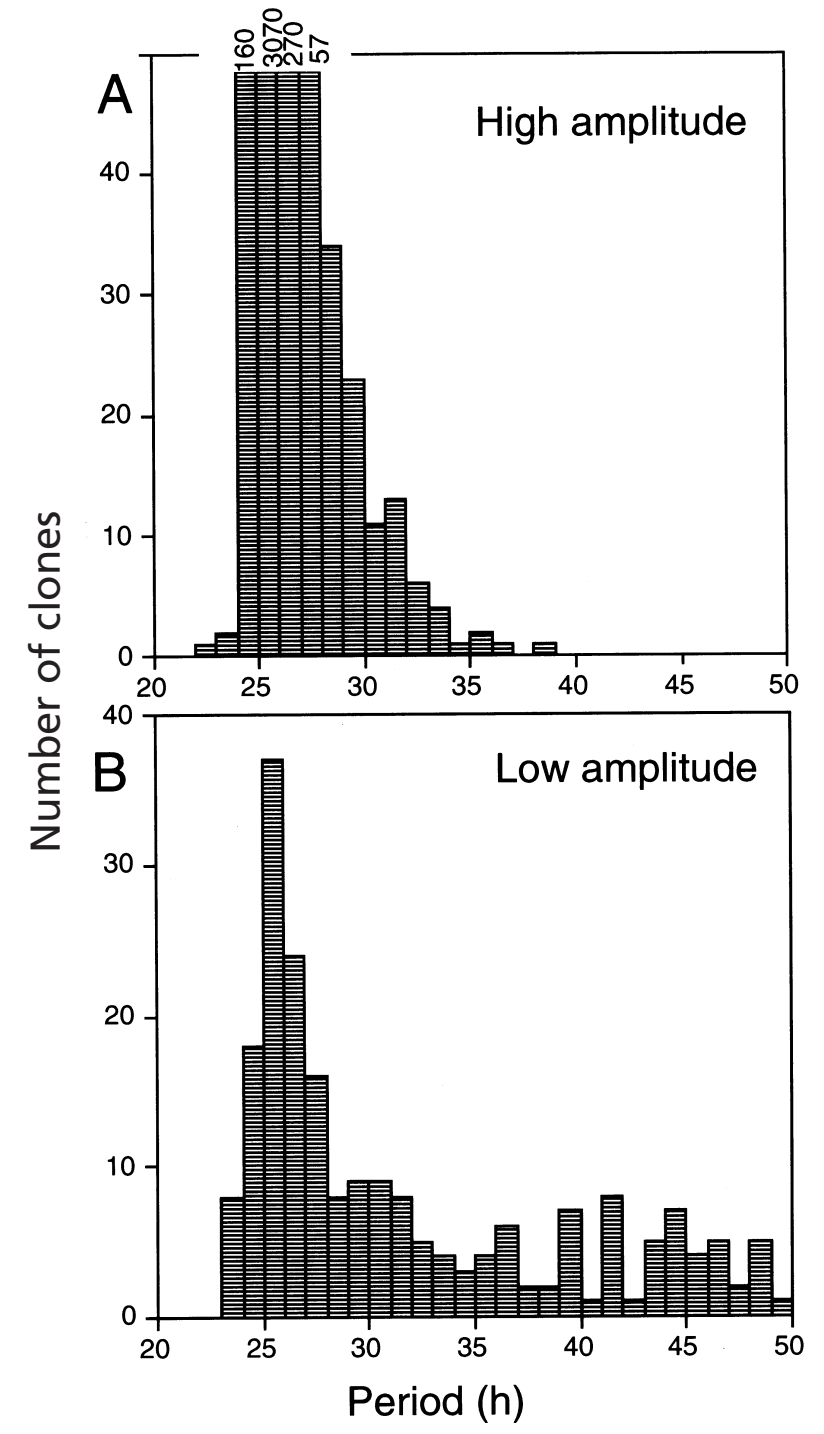

Fig. 2. Period distribution of kaiA mutants. Bioluminescence rhythms for clones that carry error-prone PCR copies of kaiA were assayed with a CCD camera system. The distribution of 3874 clone periods is shown in a histogram of $1 \mathrm{~h}$ steps. On the basis of peak-to-trough ratio of bioluminescence, these rhythms were classified as high amplitude and low amplitude/arrhythmic. (A) Histogram of the periods of 3648 high-amplitude clones (peak-to-trough ratio $>10$ ). (B) Histogram of the periods of 226 medium- and low-amplitude clones (peak-to-trough ratio $<10$ ).

trated in Fig. 4, we were able to identify in KaiA an evident cluster of period mutations (residues 239-245) and another weak one of period mutations (residues 113-119). This suggests that cluster regions of KaiA are important in controlling the period length of the circadian oscillation, since most mutations that mapped to these regions have long period phenotypes (arrhythmia-causing mutations have not been found in these regions so far). The regions of KaiA that sustain rhythm by enhancing $k a i B C$ expression are likely located elsewhere, because the mutations in arrhythmic mutants mapped to other parts of KaiA. These mutants (C53S, V76A, F178S, F224S, E274K) are also very interesting because these residues are essential for enhancing kaiBC expression. To make more detailed and reliable assessments of the different regions of KaiA, more mutations need to be identified.

Three very-long-period mutants (phenotype similar to those shown in Fig. 3D) carried double mutations in the kaiA sequence. Three other mutations that substantially affect KaiA all induced arrhythmicity. These included a missing start codon (GTG $\rightarrow$ GCG), a stop codon insertion (Q117) and a frame-shift at residue 143. The severe phenotype resulting from these mutations is also compatible with a crucial role for KaiA in sustaining the cyanobacterial circadian rhythm. Such a function could be interaction(s) of KaiA with other clock proteins (especially with KaiC, Iwasaki et al., 1999; Taniguchi et al., 2001), or alteration of phosphorylation of KaiC by KaiA (T. Nishiwaki, Y. Kitayama, H. Iwasaki \& T. Kondo and H. Iwasaki, Y. Kitayama \& T. Kondo, unpublished data).

\section{Function of KaiA}

By examining kaiBC expression of many KaiA mutants, we found that the KaiA protein could extend the period to some extent without lowering kaiBC expression. KaiA may interact with other clock proteins (especially with KaiC, Iwasaki et al., 1999; Taniguchi et al., 2001), possibly altering the phosphorylation of KaiC (Nishiwaki et al., 2000) or forming hetero-complexes of clock proteins (Iwasaki et al., 1999). KaiA function is not yet well understood; analysis of the mutants identified in this research and, in particular, identification of regions in which they cluster, will lead to further elucidation of its biochemical mechanism of action. It should be noted that mutations can enhance or diminish protein activity (e.g. enzymic or binding activity), although it is assumed generally that most random mutations lower protein activity. In contrast to mutations in $\mathrm{KaiB}$ or $\mathrm{KaiC}$, the vast majority of KaiA mutations extended the period and only rarely shortened it. The statistical observations made in this study lead us to conclude that the biochemical function of the KaiA protein is to move the clock operation forward.

On the other hand, in KaiA mutants with severe phenotypes, kaiBC expression was lowered by $40-50 \%$ in very-long-period mutants and by $90 \%$ in arrhythmic KaiA mutants compared to wild-type. As our previous study (Ishiura et al., 1998) revealed that a function of KaiA was to activate kaiBC transcription and that kaiBC expression was severely lowered in a $\mathrm{kaiA}^{-}$ strain, our observations for severe KaiA mutants are compatible with our previous study (Ishiura et al., 1998). Clearly, arrhythmic mutants obtained in this study were very important for dissection of these actions. Interestingly, the site of arrhythmic or severe lowamplitude mutants was not included in the region in which moderate-period mutants clustered. These results 


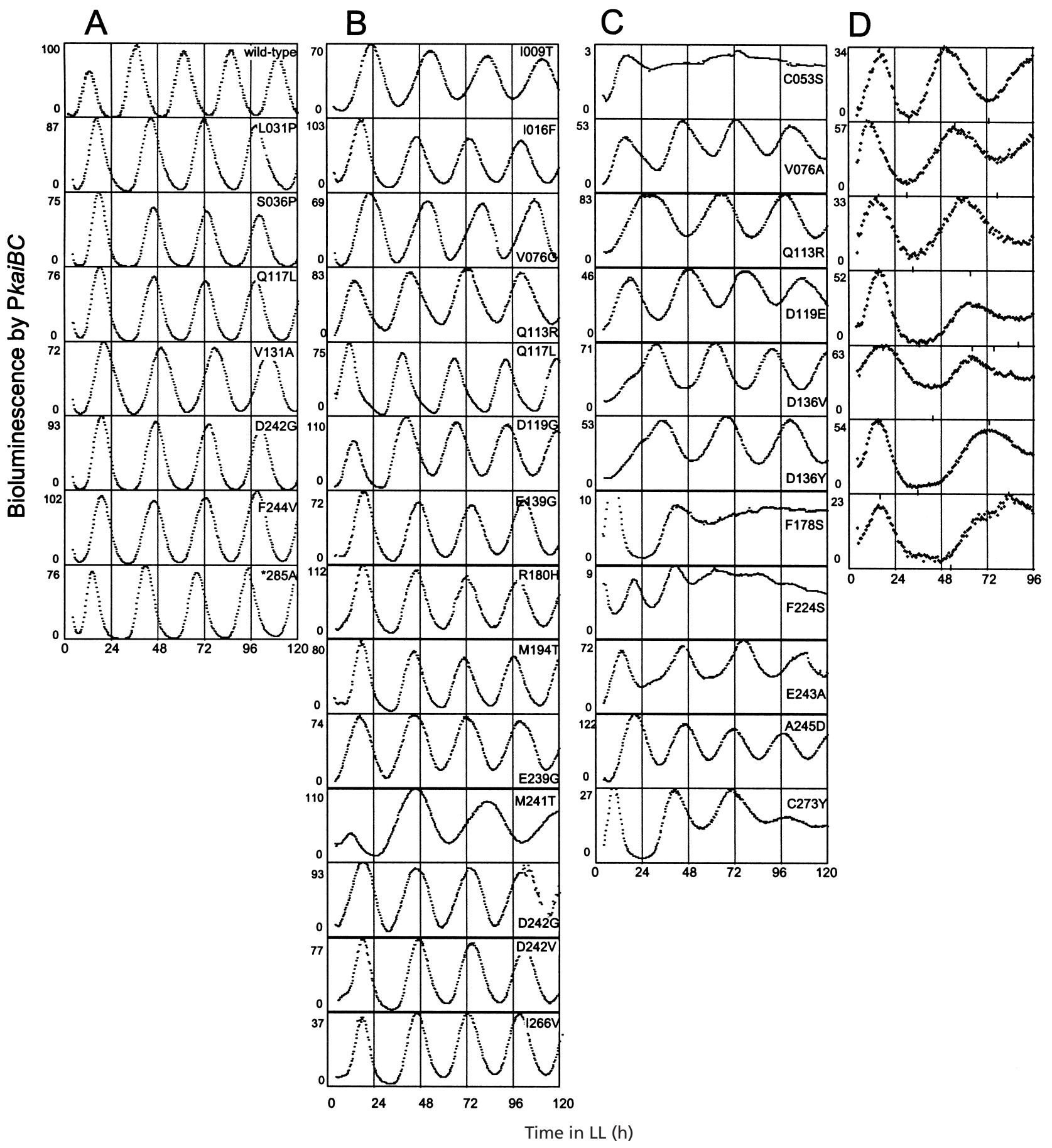

Fig. 3. Circadian bioluminescence profiles of kaiA mutants. Bioluminescence rhythms of representative mutants. After specific mutations were identified, rhythms were tracked with a PMT-based system to assay the rhythmic profile precisely. Mutants were cultured on a small dish containing BG-11 solid medium. After being cultured under LL for 3 days, clones were subject to $12 \mathrm{~h}$ darkness to reset the rhythm, then monitored using the PMT system in LL for bioluminescence. The temperature was kept at $30^{\circ} \mathrm{C}$ throughout the study. Bioluminescence intensity of each trace is normalized to that of the wild-type (NUC42) rhythm. (A) Wild-type (NUC42) and high-amplitude (peak-to-trough ratio > 10) group; (B) mediumamplitude group $(10>$ peak-to-trough ratio $>3)$; (C) low-amplitude or arrhythmic mutants (peak-to-trough ratio $<3$ ). Vertical axes are normalized to maximum bioluminescence. Bioluminescence intensities are shown on a relative scale (percentage of wild-type); (D) clones with very long periods. Vertical axes are normalized to maximum bioluminescence. These profiles were those obtained from screening with the CCD camera system. Bioluminescence of each mutant is normalized to that of the wild-type. Mutations in kaiA of these mutants have not yet been identified. 


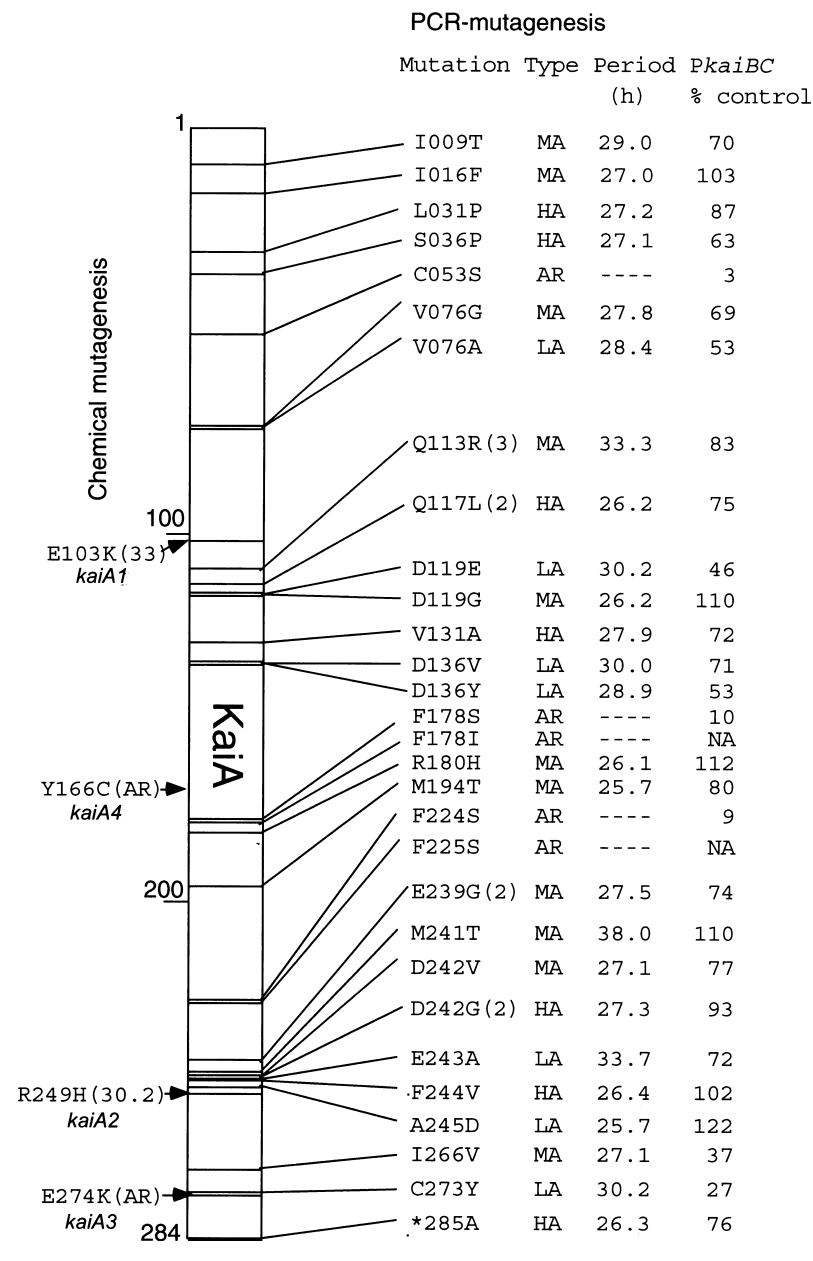

Fig. 4. Mapping of kaiA mutations onto the KaiA amino acid sequence. Point mutations, as identified by direct sequencing of each mutant DNA, were mapped onto the KaiA protein. Mutations resulting in multiple substitution or frame-shifts are not included. Phenotypes are listed in three columns. HA, MA, LA and AR in Type column represent high amplitude, medium amplitude, low amplitude and arrhythmic, respectively. The period of the rhythm in hours and PkaiBC activity (percentage of wild-type) are also listed. NA, not examined. Numbers in parentheses for Q113R, Q117L, E239G and D242G indicate duplicated clones identified by sequencing. Mutants obtained by chemical (EMS) mutagenesis are also shown on the left side of the figure. Column PkaiBC lists the maximum bioluminescence intensity attained by each mutant during an assay period of 3-4 days (normalized to that of the wild-type).

also suggest the dual action of KaiA in the circadian time-keeping process in cyanobacteria.

\section{ACKNOWLEDGEMENTS}

The authors thank Drs H. Iwasaki and T. Oyama (Nagoya University) for helpful discussion and all members of the T.K. laboratory who helped in the experiments described here. This research was partly supported by grants from the Japanese Ministry of Education, Science and Culture (11233203 and COE 13CE2005) and from the Japanese Society for Promotion of Science $(11440234,11558089)$ to T.K.

\section{REFERENCES}

Bünning, E. (1973). The Physiological Clock, 3rd edn. Heidelberg: Springer.

Bustos, S. A. \& Golden, S. S. (1991). Expression of the $p s b D I I$ gene in Synechococcus sp. strain PCC 7942 requires sequences downstream of the transcription start site. J Bacteriol 173, 7525-7533.

Diaz, J. J., Rhoads, D. D. \& Roufa, D. J. (1991). PCR-mediated chemical mutagenesis of cloned duplex DNAs. Biotechniques 11, 204-206, 208, 210-211.

Dunlap, J. C. (1999). Molecular bases for circadian clocks. Cell 96, 271-290.

Golden, S. S., Johnson, C. H. \& Kondo, T. (1998). The cyanobacterial circadian system: a clock apart. Curr Opin Microbiol 1, 669-673.

Ishiura, M., Kutsuna, S., Aoki, S., Iwasaki, H., Andersson, C. R., Tanabe, A., Golden, S. S., Johnson, C. H. \& Kondo, T. (1998). Expression of a gene cluster $k a i A B C$ as a circadian feedback process in cyanobacteria. Science 281, 1519-1523.

Iwasaki, H. \& Kondo, T. (2000). The current state and problems of circadian clock studies in cyanobacteria. Plant Cell Physiol 41, 1013-1020.

Iwasaki, H., Taniguchi, Y., Ishiura, M. \& Kondo, T. (1999). Physical interactions among circadian clock proteins KaiA, KaiB and KaiC in cyanobacteria. EMBO J 18, 1137-1145.

Iwasaki, H., Williams, S. B., Kitayama, Y., Ishiura, M., Golden, S. S. \& Kondo, T. (2000). A KaiC-interacting sensory histidine kinase, SasA, necessary to sustain robust circadian oscillation in cyanobacteria. Cell 101, 223-233.

Kondo, T. \& Ishiura, M. (2000). The circadian clock of cyanobacteria. Bioessays 22, 10-15.

Kondo, T., Strayer, C. A., Kulkarni, R. D., Taylor, W., Ishiura, M., Golden, S. S. \& Johnson, C. H. (1993). Circadian rhythms in prokaryotes: luciferase as a reporter of circadian gene expression in cyanobacteria. Proc Natl Acad Sci US A 90, 5672-5676.

Kondo, T., Tsinoremas, N. F., Golden, S. S., Johnson, C. H., Kutsuna, S. \& Ishiura, M. (1994). Circadian clock mutants of cyanobacteria. Science 266, 1233-1236.

Narusaka, Y., Narusaka, M., Satoh, K. \& Kobayashi, H. (1999). In vitro random mutagenesis of the D1 protein of the photosystem II reaction center confers phototolerance on the cyanobacterium Synechocystis sp. PCC 6803. J Biol Chem 274, 23270-23275.

Nishiwaki, T., Iwasaki, H., Ishiura, M. \& Kondo, T. (2000). Nucleotide binding and autophosphorylation of the clock protein $\mathrm{KaiC}$ as a circadian timing process of cyanobacteria. Proc Natl Acad Sci US A 97, 495-499.

Pittendrigh, C. S. (1993). Temporal organization: reflections of a Darwinian clock-watcher. Annu Rev Physiol 55, 17-54.

Prentki, P. \& Krisch, H. M. (1984). In vitro insertional mutagenesis with a selective DNA fragment Gene 29, 303-313.

Taniguchi, Y., Yamaguchi, A., Hijikata, A., Iwasaki, H., Kamagata, K., Ishiura, M., Go, M. \& Kondo, T. (2001). Two KaiA-binding domains of cyanobacterial circadian clock protein KaiC. FEBS Lett 496, 86-90.

Young, M. W. \& Kay, S. A. (2001). Time zones: a comparative genetics of circadian clocks. Nat Rev Genet 9, 702-707.

Received 7 January 2002; revised 15 May 2002; accepted 17 May 2002. 